\title{
Prediction of Mandibular Growth Pattern Using Palatoscopy: A Retrospective Model Analysis
}

\author{
Swaroop Subramonia ${ }^{1}$, Prasanna T Ramaiah ${ }^{2}$, Suhas Setty ${ }^{3}$, Mayank Khandelwal ${ }^{4}$, Chethan K Dakshina ${ }^{5}$, Cilpa Varghese ${ }^{6}$
}

\begin{abstract}
Aim and objective: To predict and correlate mandibular growth pattern using palatoscopy (average, horizontal, and vertical).

Materials and methods: This retrospective study includes 120 archived maxillary casts. One key person and two examiners performed the study; the key person was responsible for coding and collecting casts and radiographs, as well as calibration of the examiners. The key person divided the samples into three craniofacial growth pattern groups (average, horizontal, and vertical) based on Frankfort-mandibular plane angle (FMA), SN-GoGn, and facial axis angle and distributed them to the blinded examiners. Rugae were studied using a modified Thomas and Kotze classification system. After the analysis, the results were decoded and analyzed with the corresponding cephalometric radiographs. Results: Multinomial logistic regression was employed to predict the mandibular growth patterns with the following independent variables: Forwardly directed rugae, number of divergent rugae, predominant shape circular, predominant shape curved, primary rugae, predominant shape wavy, number of convergent rugae, and horizontally directed rugae.

Conclusion: Palatal rugae patterns predict the growth pattern of the mandible.

Clinical significance: Considering the significant parameters and their correlation with mandibular growth pattern, prediction of mandibular growth patterns can be done noninvasively.

Keywords: Craniofacial growth pattern, Mandibular growth pattern, Mandibular rotation, Palatoscopy.

The Journal of Contemporary Dental Practice (2021): 10.5005/jp-journals-10024-3060
\end{abstract}

\section{INTRODUCTION}

The craniofacial complex develops in a foreseeable, chronological, and arranged manner, albeit with a wide range of variations in the amount and timing of growth. Predicting mandibular growth patterns is an integral part of orthodontic treatment planning, and cephalometry is commonly used for the same. Analyzing measurements of various structures from the craniofacial complex (symphysis, frontal sinus, the body of mandible, and mandibular condyle), either individually or in an association, provides likely mandibular growth pattern predictions. Accuracy of growth pattern predictions is a challenge, despite the conclusion of various cephalometric studies done across the globe, in addition patients undergo multiple diagnostic radiation exposures., ${ }^{1,2}$

Palatal rugae are formed in the third month of intrauterine life and retain their characteristics except for an increase in size due to their normal growth. ${ }^{3}$ At birth, palatal rugae are complete, creating a unique pattern, and remain stable over a more extended period of life. The anterior rugae do not increase in length after 10 years of age $\mathrm{e}^{4}$ and are commonly used by dental clinicians as a reference point to evaluate the extent of the tooth movement. Different dental anthropometric studies have correlated relationship between palatal rugae and growth parameters like arch length, facial height, facial form, and various sagittal relationships., ${ }^{5,6}$ Due to the complexity and cumbersome nature of cephalometrics, a newer method to assess the growth pattern without ancillary radiation is needed. For this concern, palatoscopy as a method comes into the limelight.

The purpose of this hypothetical study is to assess if a relationship exists between palatal rugae and the direction of mandibular growth. Taking into consideration the stability and uniqueness of palatal rugae, it can also be used to determine the mandibular growth vectors.
1,2,4-6 Department of Orthodontics, Sri Siddhartha Dental College and Hospital, Sri Siddhartha Academy of Higher Education, Tumkur, Karnataka, India

${ }^{3}$ Department of Oral Medicine and Radiology, Siddhartha Dental College and Hospital, Sri Siddhartha Academy of Higher Education, Tumkur, Karnataka, India

Corresponding Author: Swaroop Subramonia, Department of Orthodontics, Sri Siddhartha Dental College and Hospital, Sri Siddhartha Academy of Higher Education, Tumkur, Karnataka, India

How to cite this article: Subramonia S, Ramaiah PT, Setty S, et al. Prediction of Mandibular Growth Pattern Using Palatoscopy: A Retrospective Model Analysis. J Contemp Dent Pract 2021;22(3):264-267.

Source of support: Nil

Conflict of interest: None

In this study, we have evaluated the use of palatal rugae patterns (palatoscopy) as an alternate and easy method to predict mandibular growth patterns.

\section{Materials and Methods}

This retrospective study was carried out using archived 120 maxillary (upper) casts from the record room of the Department of Orthodontics and Dentofacial Orthopedics. The study involved one key person and two examiners. The key person was responsible for collecting casts, radiographs, calibration of the examiners as well as coding of the casts wherein the Key person then divided the study casts into three craniofacial growth pattern groups (average, horizontal, and vertical) based on cephalometric analysis using Frankfort-mandibular plane angle (FMA), SN-GoGn, and facial axis angle and distributed them to the blinded examiners. 
Using a sharp graphite pencil, the rugae were delineated (Table 1) and measured with digital vernier caliper. The length of all the rugae was determined and categorized into three groups: Primary ( $>5 \mathrm{~mm})$, secondary $(2-3 \mathrm{~mm})$, and tertiary $(<2 \mathrm{~mm})$.

The shapes of individual rugae were classified ${ }^{7}$ into five primary types: Unification, curved, wavy, straight, and circular (Table 2, Fig. 1).

Wavy rugae were serpentine in shape, and rugae that showed definite continuous ring formation were classified as circular. Additionally, palatal rugae that did not fall in any of the mentioned classes, i.e., nonspecific rugae pattern, were considered for unification. Unification occurred when two rugae joined at their origin or termination. Rugae were considered diverging if two rugae had the same origin but immediately branched, whereas rugae with different origins, which joined at their lateral portions, were considered converging.

Rugae were further classified based on the direction from the mid palatine raphe, forward-directed rugae, backward-directed, and horizontal rugae $^{8}$ (Fig. 2).

Table 1: Parameters of rugae

\begin{tabular}{|c|c|c|}
\hline & & Category \\
\hline \multirow[t]{3}{*}{ Length (in mm) } & & Primary $>5$ \\
\hline & & Secondary 3-5 \\
\hline & & Fragmentary $<3$ \\
\hline \multirow[t]{6}{*}{ Shape } & Branched & Unification-convergent \\
\hline & & Unification-divergent \\
\hline & Unbranched & Curved \\
\hline & & Wavy \\
\hline & & Straight \\
\hline & & Circular \\
\hline \multirow[t]{3}{*}{ Direction } & & Forward \\
\hline & & Horizontal \\
\hline & & Backward \\
\hline
\end{tabular}

Table 2: Shape of the rugae

Modified Thomas and Kotze classification of the palatal rugae
(A) Straight
(B) Wavy
(C and D) Unification
(E) Curved
(F) Circular

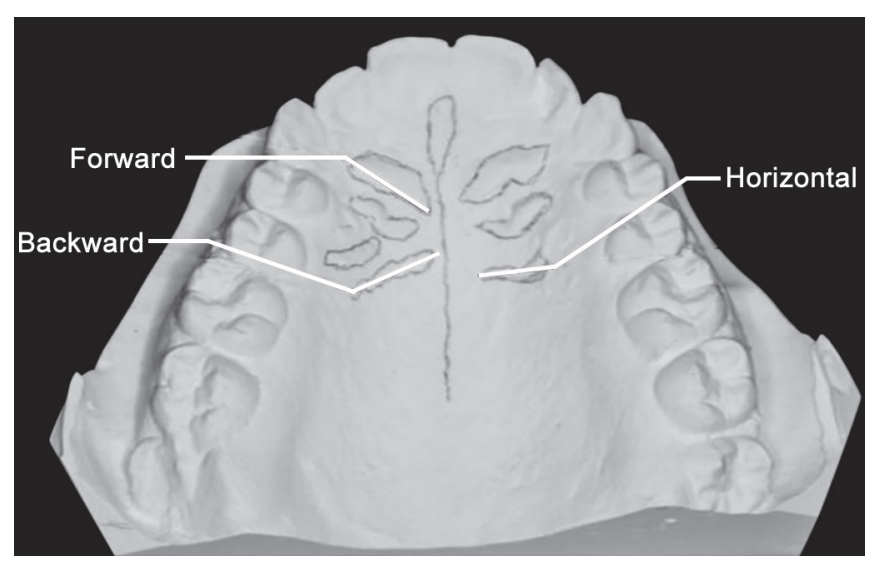

Fig. 1: Shape of the rugae

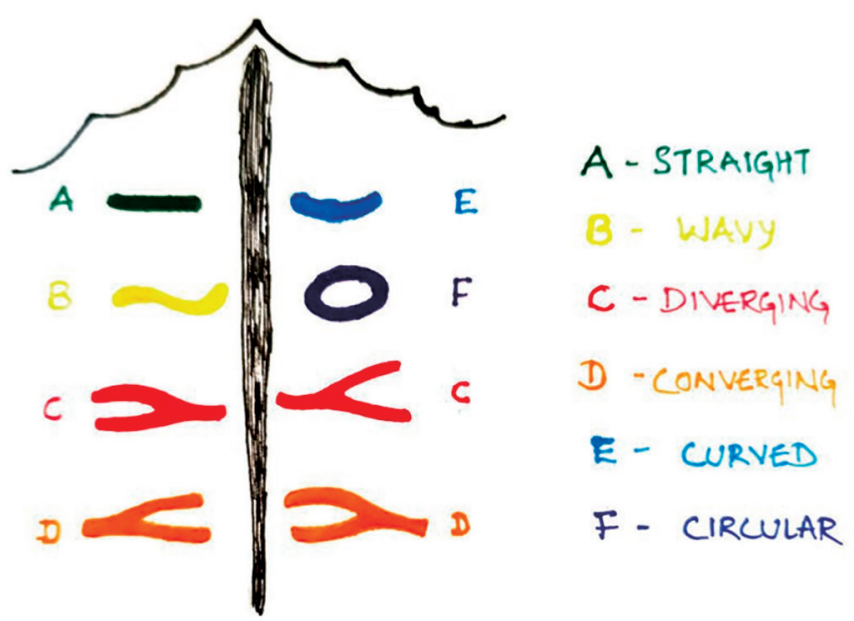

Fig. 2: Direction of the rugae

Rugae on the casts were marked to criteria by blinded investigators (examiners 1 and 2), and the results were decoded and analyzed with the corresponding cephalometric radiographs by the key person.

\section{Statistical Analysis}

Data were entered into a Microsoft Excel spreadsheet and cleaned, validated, and analyzed using SPSS (version 20). Analysis of variance test for all the parameters was applied to analyze differences, if any, between the different mandibular growth patterns. Tukey's multiple comparison test was carried out to compare the groups. Interexaminer reliability was assessed using intraclass correlation reliability analysis. Multinomial logistic regression was employed to predict the mandibular growth pattern. The level of significance was set to 0.05 (95\% confidence level).

\section{Results}

\section{Number of Rugae}

There is no significant difference between the total number of rugae and rugae on the right and left side among different mandibular growth patterns (Table 3).

\section{Length of the Rugae}

Secondary rugae were more commonly seen in the horizontal growth pattern when compared with the vertical growth pattern. However, primary rugae were less common in the horizontal growth pattern. Fragmentary rugae were equally distributed in all three growth patterns with no statistical significance (Table 3).

\section{Shape of the Rugae}

The average growth pattern group predominantly had curved rugae. The horizontal growth pattern predominantly had a wavy shape rugae (curved and circular rugae were rarely seen). The circular shape rugae were commonly seen in the vertical growth pattern (Table 3).

\section{Direction of the Rugae}

The direction of rugae showed that the horizontal growth pattern had more forwardly directed rugae, and the same was rarely seen in the average and vertical growth pattern groups. The backwardly directed rugae were commonly seen in the vertical growth pattern and were least in the horizontal growth pattern. The presence of horizontally directed rugae was highly significant in the average 
Prediction of Mandibular Growth Pattern Using Palatoscopy

Table 3: Results of multinomial logistic regression analysis predicting mandibular growth pattern

\begin{tabular}{lll}
\hline Characteristics & Horizontal, OR $(95 \% \mathrm{Cl})$ & Vertical, OR $(95 \% \mathrm{Cl})$ \\
\hline Length rugae primary & $0.369(0.215-0.634)^{* *}$ & $0.953(0.629-1.444)$ \\
Predominant shape circular & $0.679(0.300-1.538)$ & $2.387(1.211-4.706)^{*}$ \\
Predominant shape curved & $0.117(0.055-0.251)^{* *}$ & $0.379(0.204-0.707)^{* *}$ \\
Predominant shape wavy & $9.990(4.290-23.265)^{* *}$ & $8.581(4.011-18.360)^{* *}$ \\
No. of divergent rugae & $1.787(0.648-4.926)$ & $0.490(0.204-1.178)$ \\
No. of convergent rugae & $0.043(0.013-0.142)^{* *}$ & $0.281(0.125-0.634)^{* *}$ \\
Forwardly directed rugae & $5.403(2.341-12.471)^{* *}$ & $1.968(0.954-4.062)$ \\
Horizontally directed rugae & $0.650(0.281-1.505)^{* *}$ & $0.354(0.165-0.759)^{* *}$ \\
\hline
\end{tabular}

*Statistically significant at $p<0.05,{ }^{* *}$ statistically significant at $p<0.01$; Average growth pattern represents the reference category; Note: $\mathrm{OR}=$ odds ratio, $\mathrm{Cl}=$ confidence interval

growth pattern group, and the same was less frequently seen in the horizontal and vertical growth patterns (Table 3).

\section{Frequency of the Rugae}

In the horizontal growth pattern, the frequency of divergent rugae was more, whereas the vertical and average growth pattern groups displayed more of convergent rugae patterns (Table 3).

Multinomial logistic regression was employed to predict the mandibular growth patterns with the following independent variables: Forwardly directed rugae, number of divergent rugae, predominant shape (circular, curved, and wavy), primary rugae, number of convergent rugae, and horizontally directed rugae.

An increase in the value of "Primary Rugae" decreases the odds of the horizontal growth pattern by $(1-0.369=0.631) 63.31 \%$.

Multinomial regression analysis results (Table 3 ) showed the predominant shape wavy and forwardly directed rugae as the strongest predictors of the horizontal growth pattern in comparison to the average growth pattern. The predominant shape circular and predominant shape wavy are the strongest predictors of the vertical growth pattern in comparison to the average growth pattern.

A unit increase in the predominant shape wavy increases the odds of the horizontal growth pattern by 9.99 times. A unit increase in the forward direction of rugae increases the odds of the horizontal growth pattern by 5.4 times as compared to the average growth pattern.

A unit increase in the predominant shape circular increases the odds of the vertical growth pattern by 2.4 times, and a unit increase in the predominant shape wavy increases the odds of the vertical growth pattern by 8.6 times as compared to the average pattern.

An increase in the value of the number of convergent rugae decreases the odds of being horizontal pattern by $(1-0.043=0.957)$ (95.7\%).

\section{Discussion}

Orthodontic diagnosis and treatment planning encompass assessing an individual's facial skeletal pattern in all three dimensions, i.e., transverse, vertical, and sagittal. ${ }^{9,10}$ Understanding the growth pattern is very important in the early detection of developing malocclusion and to prevent relapse after correcting the malocclusion. Although all three dimensions are imperative, the vertical facial pattern forms an important aspect for orthodontic diagnosis due to its variability in treatment planning and mechanics. ${ }^{11}$ The fact that vertical facial growth, compared to other dimensions, is the least to cease, making it a crucial factor in diagnosis.

Orthodontic treatment is greatly influenced by the mandibular growth pattern. Various and extensive studies have been conducted to define mandibular rotation types, and several parameters have been used to predict the mandibular growth with wavering success. Structures like symphysis, ${ }^{12}$ frontal sinus, ${ }^{13}$ and morphological indicators within the mandible ${ }^{14-16}$ have been evaluated with the help of radiographs. FMA, SN-GoGn, Y-axis angle, facial axis angle, and Jarabak's ratio are the most commonly used parameters to predict mandibular growth patterns, but these had their own drawbacks. ${ }^{1}$

Numerous cephalometric studies have identified associated features of different types of growth patterns such as zygomatic buttress and symphysis morphology. A prominent malar bone is a common feature of the severely horizontal growth pattern in individuals. The reverse is true with vertical growth pattern in individuals. Rickets ${ }^{17}$ in his study associated a thick symphysis with an anterior growth direction, and it can be considered as one of the predictors for the direction of mandibular growth rotations.

Although various cephalometric parameters have been used to describe mandibular morphology, very few studies have reported comparison and correlation in different growth patterns.

Despite numerous cephalometric analyses, it is still very difficult to accurately predict the direction of mandibular growth. Due to its complex and cumbersome nature, studies have shown that predicting the direction of mandibular growth using cephalometric assessment is very difficult. For this concern, palatoscopy as a method for determining the mandibular growth patterns came to attention for the first time.

Taking into consideration the stability and uniqueness of palatal rugae, it can also be used to determine mandibular growth vectors. To our knowledge, no previous study has been done to assess mandibular growth patterns based on palatal rugae in dental casts.

The parameters used in this study are number, shape, length, and direction of rugae and its unification pattern. ${ }^{7,8}$ The results of interexaminer analysis showed that there was no significant difference among the results of examiners 1 and 2 . Hence, considering the results of examiners 1 and 2 and the maximum reliability among the examiners, the correlation of unification among the mandibular growth pattern has high significance. In the horizontal growth pattern, the frequency of divergent rugae was more, whereas in vertical and average growth patterns, more of convergent rugae were seen.

Correlating the number of rugae with growth patterns, the results were not statistically significant. There was also no significant correlation between the number of rugae on right and left sides of the arch relating to the mandibular growth pattern.

Considering the length of rugae, secondary rugae were more commonly seen in the horizontal growth pattern when compared to the vertical growth pattern with the $p$ value of 0.675 
Table 4: Reference chart

\begin{tabular}{lllll}
\hline SI. No. & Parameters & Horizontal & Vertical & Average \\
\hline 1 & Forwardly directed rugae & $\uparrow \uparrow$ Sig & $\downarrow$ Sig & $\downarrow$ Sig \\
2 & No. of divergent rugae & $\uparrow \uparrow$ Sig & & \\
3 & Circular rugae & $\downarrow$ Sig & $\uparrow \uparrow$ Sig & \\
4 & Curved rugae & $\downarrow$ Sig & $\uparrow$ Sig & $\uparrow \uparrow$ Sig \\
5 & Primary rugae & $\downarrow$ Sig & $\uparrow$ Sig & $\uparrow \uparrow$ Sig \\
6 & Wavy rugae & $\uparrow \uparrow$ Sig & $\uparrow$ Sig & $\downarrow$ Sig \\
7 & No. of convergent rugae & $\downarrow$ Sig & $\uparrow$ Sig & $\uparrow \uparrow$ Sig \\
8 & Horizontally directed rugae & $\uparrow$ Sig & $\downarrow$ Sig & $\uparrow \uparrow$ Sig \\
\hline
\end{tabular}

$\downarrow$, not significant (point --1 ); $\uparrow$, significant (point -+1 ); $\uparrow \uparrow$, highly significant (point -+2 )

and standard error of 0.226 . However, primary rugae were less common in the horizontal growth pattern. Fragmentary rugae were equally distributed in all three growth patterns with no statistical significance.

The shape of the rugae showed that the average growth pattern predominantly had curved shape rugae that were highly significant, whereas the curved shape rugae were rarely seen in the horizontal growth pattern. The horizontal growth pattern predominantly had wavy-shaped rugae. The circular-shaped rugae were commonly seen in the vertical growth pattern, which were rarely seen in the horizontal growth pattern.

The direction of rugae showed that the horizontal growth pattern had more forwardly directed rugae, and the same was rarely seen in the average and vertical growth patterns. The backwardly directed rugae were commonly seen in the vertical growth pattern and were least in the horizontal growth pattern. The horizontally directed rugae were commonly seen in the average growth pattern, which were highly significant. The same was less frequently seen in the horizontal and vertical growth patterns.

Considering the results obtained from the present study, it is clearly evident that palatoscopy as a method for determining the mandibular growth patterns is highly beneficial due to its ease of use, ease of interpretation, cost-effectiveness, and reduced radiation exposure.

Interpretation of palatoscopy for the prediction of mandibular growth patterns can be done by the reference chart (Table 4) given below. Considering the significant parameters, we can allocate points or scores to the individual significant parameters based on the correlation to different mandibular growth patterns, and predictions can be done.

According to the results obtained in the present study, a conclusion can be drawn that the higher the total of values designed to the eight parameters, the higher are the chances of an average growth pattern followed by the vertical growth pattern followed by the horizontal growth pattern, respectively.

But, further studies with a larger sample size are required to prove its effectiveness such that it can be extrapolated for common use.

\section{Conclusion}

Since the diagnosis, treatment planning that includes the decision to extract or not, anchorage preparation, biomechanics applied, and period of retention are dependent on different growth patterns. Therefore, the prediction of an abnormal skeletal growth pattern based on morphologic analysis can represent an important step in orthodontic diagnosis and treatment planning.

According to the results obtained in the present study, a conclusion can be drawn that the higher the points that we allocate, the higher the chances of the average growth pattern followed by the vertical growth pattern followed by the horizontal growth pattern, respectively. Hence, the palatal rugae can be used for predicting the growth rotations of the mandible.

\section{Limitations}

In our study, we have attempted to predict mandibular growth patterns using an association of rugae and mandibular growth parameters. Further studies of this kind can be strengthened by adopting model-fitting procedures and their validation.

\section{References}

1. Rizwan M, Mascarenhas R, Hussain A. Reliability of the existing vertical dysplasia indicators in assessing a definitive growth pattern. Rev Latinoam Ortodon Odontop 2011;16:1-5.

2. Asad S, Naeem S. Correlation between various vertical dysplasia assessment parameters. Pak Orthodont J 2009;1(1):28-33.

3. Hauser G, Daponte A, Roberts MJ. Palatal rugae. J Anat 1989;165: 237-249.

4. Patil MS, Patil SB, Acharya AB. Palatine rugae and their significance in clinical dentistry: a review of the literature. J Am Dent Assoc 2008;139(11):1471-1478. DOI: 10.14219/jada.archive.2008.0072.

5. Van der Linden FP. Changes in the position of posterior teeth in relation to ruga points. Am J Orthod 1978;74(2):142-161. DOI: 10.1016/0002-9416(78)90081-7.

6. Kallianpur S, Desai A, Kasetty $S$, et al. An anthropometric analysis of facial height, arch length, and palatal rugae in the Indian and Nepalese population. J Forensic Dent Sci 2011;3(1):33-37. DOI: 10.4103/0975-1475.85294.

7. Thomas $C$, Kotze T. The palatal ruga pattern in six southern African human populations. Part I: a description of the populations and a method for its investigation. J Dent Assoc S Afr 1983;38:158-165.

8. Kapali $S$, Townsend G, Richards $L$, et al. Palatal rugae patterns in Australian aborigines and Caucasians. Aust Dent J 1997;42(2):129-133. DOI: 10.1111/j.1834-7819.1997.tb00110.x.

9. Hirschfeld W, Moyers R. Prediction of craniofacial growth. The state of the art. Am J Orthod 1971;60(5):435-444. DOI: 10.1016/00029416(71)90112-6.

10. Nathani R, Diagavane P, Shrivastav S, et al. Evaluation of frontal sinus as a growth predictor in horizontal, vertical, and average growth pattern in children from 8 to 11 years: a cephalometric study. J Indian Orthod Soc 2016;50(2):101-105. DOI: 10.4103/0301-5742.179944.

11. Ahmed M, Shaikh A, Fida M. Diagnostic performance of various cephalometric parameters for the assessment of vertical growth pattern. Dental Press J Orthod 2016;21(4):41-49. DOI: 10.1590/21776709.21.4.041-049.oar.

12. Aki T, Nanda R, Currier F, et al. Assessment of symphysis morphology as a predictor of the direction of mandibular growth. Am J Orthod Dentofacial Orthop 1994;106(1):60-69. DOI: 10.1016/S08895406(94)70022-2.

13. Rossouw PE, Lombard CJ, Harris AM. The frontal sinus and mandibular growth prediction. Am J Orthod Dentofacial Orthop 1991;100(6): 542-546. DOI: 10.1016/0889-5406(91)70095-E.

14. Leslie LR, Southard TE, Southard KA, et al. Prediction of mandibular growth rotation assessment of the Skieller, Björk, and Linde-Hansen method. Am J Orthod Dentofacial Orthop 1998;114(6):659-667. DOI: 10.1016/s0889-5406(98)70198-2.

15. Björk A. The use of metallic implants in the study of facial growth in children: method and application. Am J Phys Anthropol 1968;29(2):243-254. DOI: 10.1002/ajpa.1330290217.

16. Davidovitch M, Eleftheriadi I, Kostaki A, et al. The use of Bjork's indications of growth for evaluation of extremes of skeletal morphology. Eur J Orthod 2016;38(6):555-562. DOI: 10.1093/ejo/ cjv084.

17. Ricketts R. Cephalometric synthesis. Am J Orthod 1960;46(9):647-673. DOI: $10.1016 / 0002-9416(60) 90172-X$. 